\author{
В.Е. Сюткин ${ }^{1, *}$, А.О. Буеверов ${ }^{2,3}$, П.О. Богомолов ${ }^{3}$ \\ 'ГБУЗ «Научно-исследовательский институт скорой помощи имени Н.В. Склифосовского», \\ Департамента здравоохранения города Москвы, Москва, Российская Федерация \\ ${ }^{2}$ ФГАОУ ВО «Первый Московский государственный медицинский университет им. И.М. Сеченова» \\ (Сеченовский Университет) Министерства здравоохранения Российской Федерации, Москва, Российская Федерация \\ ${ }^{3}$ ГБУз Московской области «Московский областной научно-исследовательский клинический институт \\ им. М.Ф. Владимирского», Москва, Российская Федерация
}

Булевиртид - новый противовирусный препарат, одобренный для лечения хронического гепатита D, оптимальный режим назначения которого требует уточнения.

Цель: сравнить эффективность монотерапии булевиртидом и комбинированного лечения булевиртидом и пегилированным интерфероном (ПЕГ-ИФН) на основании опубликованных результатов исследований.

Основные положения: на основании анализа результатов исследований MYR201 и MYR203 можно сделать вывод о более частом исчезновении HBsAg и PHK HDV, а также более выраженном снижении виремии HDV при комбинированной терапии булевиртидом и ПЕГ-ИФН, чем при монотерапии булевиртидом.

Заключение. Комбинированная терапия ПЕГ-ИФН и булевиртидом имеет ряд преимуществ перед монотерапией булевиртидом у больных хроническим гепатитом D. Требуются дальнейшие исследования для уточнения оптимальной дозы булевиртида и необходимой продолжительности терапии.

Ключевые слова: вирус гепатита D, хронический гепатит D, булевиртид, пегилированный интерферон Конфликт интересов: автор заявляет об отсутствии конфликта интересов.

Для цитирования: Сюткин В.Е., Буеверов А.О., Богомолов П.О. Имеет ли преимущество комбинированный режим лечения хронического гепатита D пегилированным интерфероном и булевиртидом перед монотерапией булевиртидом? Российский журнал гастроэнтерологии, гепатологии, колопроктологии. 2020;30(4):23-27. https://doi.org/10.22416/13824376-2020-30-4-23-27.

\title{
Is Combined Bulevirtide and Pegylated Interferon Therapy for Chronic Hepatitis D Superior over Bulevirtide Monotherapy?
}

\footnotetext{
Vladimir E. Syutkin ${ }^{1, \star}$, Alexey O. Bueverov ${ }^{2,3}$, Pavel O. Bogomolov ${ }^{3}$

${ }^{1}$ Sklifosovsky Clinical and Research Institute for Emergency Medicine, Moscow, Russian Federation

2 Sechenov First Moscow State Medical University (Sechenov University), Moscow, Russian Federation

${ }^{3}$ Vladimirsky Moscow Regional Research and Clinical Institute, Moscow, Russian Federation
}

Bulevirtide is a novel antiviral agent approved for suppression of chronic hepatitis $D$ but requiring further research into optimising the treatment scheme.

Aim. Comparative assessment of bulevirtide monotherapy versus combined treatment with bulevirtide and pegylated interferon (PEG-IFN) using published trial data.

Key points. MYR201 and MYR203 trials expose a higher frequency of HBsAg and HDV RNA extinction, as well as more effective HDV suppression for combined bulevirtide/PEG-IFN therapy compared to bulevirtide monotherapy. 
Conclusion. Combined bulevirtide/PEG-IFN therapy has particular advantages over bulevirtide monotherapy in patients with chronic hepatitis $\mathrm{D}$. Further research is required to optimise bulevirtide dosage and duration of therapy. Keywords: hepatitis D virus, chronic hepatitis D, bulevirtide, pegylated interferon Conflict of interest: the author declares no conflict of interest.

For citation: Syutkin V.E., Bueverov A.O., Bogomolov P.O. Is Combined Bulevirtide and Pegylated Interferon Therapy for Chronic Hepatitis D Superior over Bulevirtide Monotherapy? Gastroenterology, Hepatology, Coloproctology. 2020;30(4):23-27. https://doi. org/10.22416/1382-4376-2020-30-4-23-27.

Открытие вируса гепатита D (HDV) в середине 70-х годов прошлого века было связано с выявлением нового антигена в ядрах гепатоцитов у больных тяжелыми формами гепатита В. Вирион состоит из рибонуклеопротеина, включающего $\mathrm{HD}-\mathrm{Ag}$ и PHK HDV, который заключен в белковые структуры поверхностного антигена HBV (HBsAg) [1]. Вирус гепатита D является наименьшим из содержащих РНК вирусов животных, репликация которого зависит от ферментных систем гепатоцита хозяина, а сборка вириона - от белковых структур HBV [2].

До недавнего времени возможности противовирусной терапии (ПВТ) гепатита D были ограничены применением препаратов стандартного и пегилированного интерферона альфа (ПЕГ-ИФН). Трудности разработки специфической терапии HDV связаны как с отсутствием вирусных ферментных мишеней (протеаза, полимераза), так и со сложностью оценки эффективности терапии (необходимостью учета маркеров одновременно двух инфекций) [2].

Поскольку HBV необходим для сборки и межклеточного распространения HDV, инфекция HDV непосредственно связана с инфекцией HBV. Именно на этом основана разработка и первый опыт клинического применения препарата булевиртид, представляющего собой ингибитор проникновения HDV и HBV в клетку. Это синтетический полипептид, состоящий из 47 аминокислот PreS1 области L-HBsAg с присоединенным остатком миристиновой кислоты. Структура булевиртида копирует участок оболочечного белка HBV, который взаимодействует с натрий-таурохолат котранспортирующим полипептидом (NTCP), белком-транспортером солей желчных кислот, располагающимся на мембране гепатоцитов. Мимикрируя под белок вирусной оболочки, булевиртид присоединяется к NTCP и блокирует проникновение HBV и вместе с ним - HDV в гепатоцит [2, 3].

Цель: сравнить эффективность монотерапии булевиртидом и комбинированного лечения булевиртидом и ПЕГ-ИФН на основании опубликованных результатов исследований.

В нашем распоряжении для анализа имеются результаты трех клинических испытаний булевиртида, охватывающих в общей сложности 202 пациента, из которых булевиртид получал 151 пациент. Результаты исследования MYR201 опубликованы в полнотекстовом формате [4], результаты двух других исследований (MYR202 и MYR203) опубликованы в виде абстрактов [5, 6]. Кроме того, результаты исследований MYR201 (NCT02637999) и MYR202 (NCT03546621) доступны на сайте clinicaltrials.gov, содержащем информацию о клинических исследованиях по всему миру. К сожалению, результаты исследования MYR202, в которое включено наибольшее (118) число пациентов, не будут полезны для ответа на обсуждаемый вопрос, поскольку в этом исследовании не изучена комбинированная терапия булевиртидом и ПЕГИФН.

Статистическая обработка данных проведена с использованием программного пакета Statistica 8.0 (StatSoft). Для сравнения пропорций применен критерий хи-квадрат $\left(\chi^{2}\right)$.

Исчезновение HBsAg не было достигнуто в исследованиях MYR201 и MYR202, в которых больные получали терапию булевиртидом в течение 24 недель. В исследовании MYR203, в котором продолжительность терапии булевиртидом составила 48 недель, исчезновение HBsAg по завершении 24-недельного периода наблюдения имело место у 4 из 30 больных, получавших комбинированную терапию пегилированным интерфероном и булевиртидом, и ни в одном случае у больных, получавших монотерапию одним из этих препаратов $\left(\chi^{2}=2,2 ; p=0,14\right)[6]$.

Снижение содержания РНК HDV в крови в 10 и более раз от исходного уровня на 24-й неделе терапии наблюдалось у 7 из 8 пациентов $(87,5 \%)$, получавших комбинированную терапию ПЕГИФН и булевиртидом, и такого же числа пациентов, получавших монотерапию булевиртидом, в исследовании MYR201 в зависимости от назначенной ПВТ [7]. При анализе, проведенном в зависимости от фактически полученного лечения, необходимо учитывать, что у одного из больных, рандомизированных в группу монотерапии булевиртидом, перед началом лечения PHК HDV в крови не выявлялась. Один пациент выбыл из группы комбинированной терапии до 12-й недели из-за нежелательных явлений [4]. То есть снижение содержания PHК HDV в крови в 10 и более раз от исходного уровня на 24-й неделе монотерапии булевиртидом наблюдалось у $6(85,7 \%)$ из 7 пациентов, причем у 2 больных PHК HDV перестала выявляться в крови. Аналогичное снижение содержания РНК HDV имело место у всех 7 пациентов, получавших комбинированную терапию, но PHК HDV пере- 
стала выявляться у 5 из них $\left(\chi^{2}=2,57 ; p=0,1\right)$. Среднее снижение содержания РНК HDV в группе больных, получавших монотерапию булевиртидом, составило -1,67 $\log _{10}$ копий/мл от исходного уровня; в группе больных, получавших комбинированную терапию, $-2,59 \log _{10}$ копий/мл от исходного уровня.

На момент завершения ПВТ (48-я неделя) в группах больных, получавших комбинированную терапию, РНК HDV перестала выявляться у 15 из 30 пациентов; в группе больных, получавших монотерапию булевиртидом, - у 2 из 15 пациентов $\left(\chi^{2}=5,72 ; p=0,017\right)$. Этот эффект сохранялся и через 24 недели после завершения ПВТ (на 72-й неделе исследования - первичная конечная точка). Содержание РНК HDV в крови оставалось ниже порога чувствительности (10 ME/мл) у 12 из 30 пациентов, получавших комбинированную ПВТ, и ни в одном случае в группе больных, получавших монотерапию булевиртидом $\left(\chi^{2}=8,18\right.$; $p=0,004)[6]$.

Кроме того, через 48 недель лечения снижение медианы содержания HDV PHК по сравнению с исходным было более значительным в группах больных, получавших комбинированную терапию $\left(-5,21\right.$ и $\left.-6,13 \log _{10} \mathrm{ME} / \mathrm{мл}\right)$, по сравнению с группой больных, получавших только булевиртид $\left(-2,84 \log _{10} \mathrm{ME} /\right.$ мл).

В исследовании MYR201 активность АЛТ к 24-й неделе ПВТ нормализовалась у 6 из 8 пациентов, получавших монотерапию булевиртидом, и у 1 из 7 пациентов, получавших комбинированное лечение $\left(\chi^{2}=5,53 ; p=0,019\right)$.

В исследовании MYR203 нормализация активности АЛТ на момент завершения ПВТ (48я неделя) наблюдалась у 10 (67\%) из 15 пациентов, получавших монотерапию булевиртидом, и у $11(37 \%)$ из 30 больных, получавших комбинированное лечение $\left(\chi^{2}=3,62 ; p=0,057\right)$. По завершении периода наблюдения тенденция менялась на противоположную, хотя различия между группами не достигали уровня статистической значимости. Нормальная активность АЛТ сохранялась только у 3 (20\%) больных, получавших монотерапию булевиртидом, и у 12 (40\%) из 30 пациентов в группах комбинированной терапии $\left(\chi^{2}=1,8 ; p=0,18\right)$.

Булевиртид является новым препаратом, предложенным для лечения хронического гепатита D как в режиме монотерапии, так и в комбинации с пегилированным интерфероном. К настоящему времени применение булевиртида ограничивалось рамками клинических испытаний, опыт применения в рутинной клинической практике отсутствует. В публикациях, посвященных результатам исследований булевиртида, показано преимущество этого препарата по сравнению с монотерапией ПЕГ-ИФН альфа, который до недавнего времени оставался стандартом ПВТ хронического гепатита D. Также в исследовании MYR202 показана более высокая эффективность булевиртида по сравнению с монотерапией тенофовиром [5]. В настоящее время булевиртид зарегистрирован для клинического применения в России, и появилась необходимость сравнить эффективность этого препарата в монотерапевтическом и комбинированном с ПЕГ-ИФН режимах.

Сложность в оценке эффективности ПВТ в отношении инфекции HDV и хронического гепатита D связана с несколькими факторами. Во-первых, отсутствуют общепринятые критерии оценки эффективности терапии. Из общетерапевтических соображений, очевидно, следует обращать внимание на активность АЛТ и АСТ, выраженность фиброза печени. С вирусологических позиций важными представляются снижение содержания PHK HDV в крови и уменьшение (исчезновение) HBsAg с последующим появлением антител.

Экспертами Международного сотрудничества по гепатиту D (HDIN) в партнерстве c EASL были определены конечные точки лечения XГD в клинических исследованиях новых препаратов, при достижении которых препараты могут рассматриваться как кандидаты для условного одобрения применения в клинической практике. Идеальной конечной точкой является исчезновение HBsAg из крови (так называемое функциональное излечение), достижимой целью - неопределяемый уровень РНК HDV во время лечения и после завершения лечения. Также рекомендуется учитывать такой критерий эффективности терапии, как снижение содержания PHК HDV в крови в 100 раз и более $\left(\geq 2 \log _{10} \mathrm{ME} /\right.$ мл) по сравнению с исходным уровнем, во время лечения и после завершения лечения [8]. Осложняет анализ и различный дизайн исследований с разбивкой больных на малочисленные группы, а также отсутствие публикации полных результатов исследования MYR203.

C учетом всех этих ограничений мы не можем сделать окончательный вывод в отношении преимущества монотерапии или комбинированной терапии булевиртидом и ПЕГ-ИФН. Мы можем лишь попытаться выявить некоторые тенденции в применении этой терапии. Так, $13 \%$ случаев исчезновения HBsAg в течение 48 недель комбинированного лечения является одной из самых высоких цифр, когда-либо сообщавшихся по результатам ПВТ у больных с хроническим гепатитом В и D [9]. Тенденция к преимуществу комбинированной ПВТ по сравнению с монотерапией булевиртидом прослеживается и при анализе снижения РНК HDV в процессе и в результате лечения. При лечении хронического гепатита B (и D) препаратами ПЕГ-ИФН нередко отсутствует нормализация активности АЛТ и АСТ, а в ряде случаев даже отмечается ее увеличение. Поэтому более редкое достижение биохимического ответа на фоне комбинированной ПВТ по сравнению с монотерапией булевиртидом нам не представляется проблемным. 
Мы не проводили сравнения безопасности терапии, поскольку очевидно, что в группе комбинированной терапии будут наблюдаться все присущие препаратам ПЕГ-ИФН нежелательные явления. Эти явления хорошо изучены и могут быть корригированы в процессе ПВТ.

Остается также значительное число нерешенных вопросов, таких как необходимость стандартизации количественных методов определения РНК $\mathrm{HDV}$, подтверждение правомерности использования количественного определения виремии $\mathrm{HDV}$ в качестве суррогатной конечной точки оценки эффективности терапии, определение оптимальной длительности терапии. Необходимо уточнение исходных, промежуточных (после начала лечения) прогностических факторов, новых показателей оценки эффективности терапии.

\section{Заключение}

Комбинированная терапия ПЕГ-ИФН и булевиртидом имеет ряд преимуществ перед монотерапией булевиртидом у больных хроническим гепатитом D. Она должна рассматриваться как терапия первой линии во всех случаях, когда отсутствуют противопоказания к применению ПЕГ-ИФН. Требуются дальнейшие исследования для уточнения оптимальной дозы булевиртида и необходимой продолжительности терапии.

\section{Литература / References}

1. Rizzetto M., Hoyer B., Canese M.G., Shih J.W., Purcell R.H., Gerin J.L. Delta Agent: association of delta antigen with hepatitis B surface antigen and RNA in serum of delta-infected chimpanzees. Proc Natl Acad Sci USA. 1980;77(10):6124-8. DOI: 10.1073/pnas.77.10.6124

\section{Сведения об авторах}

Сюткин Владимир Евгеньевич* - доктор медицинских наук, ведущий научный сотрудник отделения трансплантации печени ГБУЗ «Научно-исследовательский институт скорой помощи имени Н.В. Склифосовского Департамента здравоохранения города Москвы».

Контактная информация: vladsyutkin@gmail.com;

129010 , г. Москва, Большая Сухаревская площадь, 3.

ORCID: https://orcid.org/0000-0001-8391-5211

Буеверов Алексей Олегович - доктор медицинских наук, профессор, профессор кафедры медико-социальной экспертизы, неотложной и поликлинической терапии Института профессионального образования ФГАОУ ВО «Первый Московский государственный медицинский университет имени И.М. Сеченова» (Сеченовский Университет) Министерства здравоохранения Российской Федерации, ведущий научный сотрудник отделения гепатологии ГБУЗ Московской области «Московский областной научно-исследовательский клинический институт имени М.Ф. Владимирского».

Контактная информация: bcl72@yandex.ru;

119991, г. Москва, ул. Трубецкая, д. 8, стр. 2.

ORCID: https://orcid.org/0000-0002-5041-3466
2. Mentha N., Clément S., Negro F., Alfaiate D. A review on hepatitis D: From virology to new therapies. J Adv Res. 2019;17:3-15. DOI: 10.1016/j.jare.2019.03.009

3. Yan H., Peng B., Liu Y., Xu G., He W., Ren B., et al. Viral entry of hepatitis B and D viruses and bile salts transportation share common molecular determinants on sodium taurocholate cotransporting polypeptide. J Virol. 2014;88(6):3273-84. DOI: 10.1128/JVI.03478-13

4. Bogomolov P., Alexandrov A., Voronkova N., Macievich M., Kokina K., Petrachenkova M., et al. Treatment of chronic hepatitis D with the entry inhibitor myrcludex B: First results of a phase Ib/IIa study. J Hepatol. 2016;65(3):490-8. DOI: 10.1016/j.jhep.2016.04.016

5. Wedemeyer H., Bogomolov P., Blank A., Allweiss L., Dandri-Petersen M., Bremer B., et al. Final results of a multicenter, open-label phase $2 \mathrm{~b}$ clinical trial to assess safety and efficacy of Myrcludex $\mathrm{B}$ in combination with Tenofovir in patients with chronic $\mathrm{HBV} / \mathrm{HDV}$ co-infection. Program and abstracts of the International Liver Congress $^{\mathrm{TM}} 2018$ - 53rd annual meeting of the European Association for the Study of the Liver. 2018. Paris, France. GS-005. J Hepatol. 2018;68(Suppl. 1):S3.

6. Wedemeyer H., Schöneweis K., Bogomolov P., Voronkova N., Chulanov V., Stepanova T., et al. Final results of a multicenter, open-label phase 2 clinical trial (MYR203) to assess safety and efficacy of myrcludex $\mathrm{B}$ in combination with PEG-interferon Alpha 2a in patients with chronic $\mathrm{HBV} / \mathrm{HDV}$ co-infection. Program and abstracts of the The International Liver Congress ${ }^{\text {TM }} 2019$ - 54rd annual meeting of the European Association for the Study of the Liver. 2019. Vienna, Austria GS-13. J Hepatol. 2019;70(Supp. 1):e81.7. https://clinicaltrials.gov/ct2/ show $/$ results $/$ NCT02637999? term $=$ NCT02637999\& $r a n k=$ $1 \&$ view $=$ results

8. Yurdaydin C., Abbas Z., Buti M., Cornberg M., Esteban R., Etzion O., et al. Treating chronic hepatitis delta: The need for surrogate markers of treatment efficacy. J Hepatol. 2019;70(5):1008-15. DOI: 10.1016/j. jhep.2018.12.022

9. Qiu K., Liu B., Li S.Y., Li H., Chen Z.W., Luo A.R., et al. Systematic review with meta-analysis: combination treatment of regimens based on pegylated interferon for chronic hepatitis B focusing on hepatitis B surface antigen clearance. Aliment Pharmacol Ther. 2018;47(10):1340-8. DOI: $10.1111 /$ apt.14629

\section{Information about the authors}

Vladimir E. Syutkin* - Dr. Sci. (Med.), Leading Researcher, Department of Liver Transplantation, Sklifosovsky Clinical and Research Institute for Emergency Medicine.

Contact information: vladsyutkin@gmail.com;

129010, Moscow, Bolshaya Sukharevskaya sq., 3.

ORCID 0000-0001-8391-5211

Alexey O. Bueverov - Dr. Sci. (Med.), Prof., Department of Medical and Social Expertise, Emergency and Outpatient Medicine, Institute of Vocational Education, Sechenov First Moscow State Medical University (Sechenov University); Leading Researcher, Department of Hepatology, Vladimirsky Moscow Regional Research and Clinical Institute.

Contact information: bcl72@yandex.ru; 119991, Moscow, Trubetskaya str., 8, build. 2. ORCID 0000-0002-5041-3466 
Богомолов Павел Олегович - кандидат медицинских наук, руководитель отделения гепатологии ГБУЗ Московской области «Московский областной научно-исследовательский клинический институт имени М.Ф. Владимирского».

Контактная информация: Bpo73@list.ru;

129110, г. Москва, ул. Щепкина, д. 61/2.

ORCID: https://orcid.org/0000-0003-2346-1216
Pavel O. Bogomolov - Cand. Sci. (Med.), Head of the Department of Hepatology, Vladimirsky Moscow Regional Research and Clinical Institute.

Contact information: Bpo73@list.ru;

129110, Moscow, Schepkina str., 61/2

ORCID 0000-0003-2346-1216.

Поступила: 03.06.2020 Принята: 08.07.2020 Опубликована: 30.08.2020 Submitted: 03.06.2020 Accepted: 08.07.2020 Published: 30.08.2020

* Автор, ответственный за переписку / Corresponding author 